\title{
Nivel de conocimiento del manejo estomatológico por COVID-19 en cirujanos dentistas
}

\author{
Level of knowledge about stomatological management in the context \\ of the COVID-19 pandemic in dental surgeons
}

Tania Lizeth Calizaya Chambilla ${ }^{1 a}$ Yury Miguel Tenorio Cahuana ${ }^{2 b}$ https://orcid.org/0000-0002-7959-3253

https://orcid.org/0000-0001-8679-2050

Correspondencia: ytenorioc@unjbg.edu.pe

\section{Resumen}

El objetivo de este estudio fue evaluar el nivel de conocimiento del manejo estomatológico por COVID-19 en cirujanos dentistas de Tacna, 2020. Metodología: La investigación es de tipo descriptivo, transversal. El estudio se realizó con una muestra de 165 cirujanos dentistas de Tacna, aplicando un cuestionario de conocimiento sobre el manejo estomatológico por COVID-19 basado en la Directiva Sanitaria N.$^{\circ} 100$ del Ministerio de Salud del Perú. El cuestionario cumple con los requisitos esenciales de validez y confiabilidad exigidos para el presente estudio. Resultados: Se obtuvo, como resultados, que la mayoría de cirujanos dentistas presentaron un nivel bueno con $52.36 \%$, seguidamente un nivel muy bueno con $42.42 \%$, en tercer lugar, un nivel regular con $4.85 \%$ y, finalmente, no se encontró ningún encuestado con un nivel de conocimiento malo. Conclusiones: El nivel de conocimiento de los cirujanos dentistas de Tacna es bueno. No se encontraron diferencias en el nivel de conocimiento del manejo estomatológico por COVID-19, en cuanto al sexo ni a la edad de los cirujanos dentistas de Tacna 2020.

Palabras clave: conocimiento, pandemia, COVID-19

\begin{abstract}
The objective of this study was to evaluate the level of knowledge about stomatological management in the context of the COVID-19 pandemic in dental surgeons of Tacna, 2020. Methodology: The research is descriptive, transversal. The study was carried out with a sample of 165 dental surgeons from Tacna, applying a knowledge questionnaire on stomatological management in the context of COVID-19, based on Health Directive N. ${ }^{\circ} 100$ of the Ministry of Health of Perú. The questionnaire meets the essential validity and reliability requirements for the present study. Results: It was obtained that the majority of dental surgeons presented a good level with $52.36 \%$, then a very good level with $42.42 \%$, in third place, a regular level with $4.85 \%$ and finally no respondents with a bad level of knowledge. Conclusions: The level of knowledge of dental surgeons in Tacna is good. No differences were found in the level of knowledge of stomatological management in the context of the COVID-19 pandemic, in terms of sex or age of the dental surgeons of Tacna 2020.
\end{abstract}

Keywords: knowledge, pandemic, COVID-19

\footnotetext{
${ }^{1}$ Investigador Independiente. Tacna, Perú

2 Universidad Nacional Jorge Basadre Grohmann. Escuela Profesional de Odontología. Tacna, Perú

${ }^{a}$ Cirujano Dentista

${ }^{\mathrm{b}}$ Especialista en Ortodoncia y Ortopedia Maxilar
} 


\section{Introducción}

En diciembre del 2019 se identificó el primer caso de neumonía por un nuevo virus en la ciudad de Wuhan, China. Este virus fue identificado como coronavirus síndrome respiratorio agudo grave 2 (SARS-CoV-2), siendo el causante de la enfermedad denominada por la Organización Mundial de la Salud como COVID-19. El 11 de marzo de 2020, el Director General de la OMS declaró que la enfermedad por COVID-19 era una pandemia. ${ }^{1}$ Si bien la mayor fuente de transmisión son los pacientes sintomáticos con COVID-19, se sabe, ahora, que también los pacientes asintomáticos, y en periodo de incubación, pueden transmitirlo, hecho que hace extremadamente difícil su control. ${ }^{2}$ El cirujano dentista tiene un alto riesgo de contagio debido a la naturaleza de los procedimientos y tratamientos dentales. ${ }^{3}$

Teniendo como finalidad contribuir a la reducción del impacto de la COVID-19 en la atención estomatológica del territorio nacional ${ }^{4}$ surge la siguiente interrogante $¿$ Cuál es el nivel de conocimiento del manejo estomatológico por COVID-19 en los cirujanos dentistas de Tacna? Más aún, tomando en cuenta que estamos en pleno desarrollo de esta pandemia.

\section{Materiales y métodos}

La técnica de recolección de datos fue la encuesta; y el instrumento de medición, un cuestionario de conocimiento sobre manejo estomatológico por COVID-19 compuesto por un total de 20 preguntas con respuestas dicotómicas, el cual distribuye sus preguntas en cinco dimensiones de conocimientos relacionados con los siguientes aspectos:

- Disposiciones para el establecimiento de la cita.

- Disposiciones para la sala de espera y S.S.H.H. del Servicio de Estomatología.

- Disposiciones para protección del personal de atención estomatológica y pacientes.

- Preparación del campo clínico.

- Disposiciones para el manejo de procedimientos estomatológicos.

La recolección de datos estuvo a cargo del investigador. Se visitó consultorios odontológicos privados de la ciudad de Tacna que atendían al público. En la entrevista con el cirujano dentista se le explicó los objetivos del estudio y la importancia de este; mediante su consentimiento informado se aplicó la encuesta correspondiente.

\section{Resultados}

\section{Figura 1}

Distribución sobre el nivel de conocimiento del manejo estomatológico por COVID-19 de cirujanos dentistas de Tacna (2020)

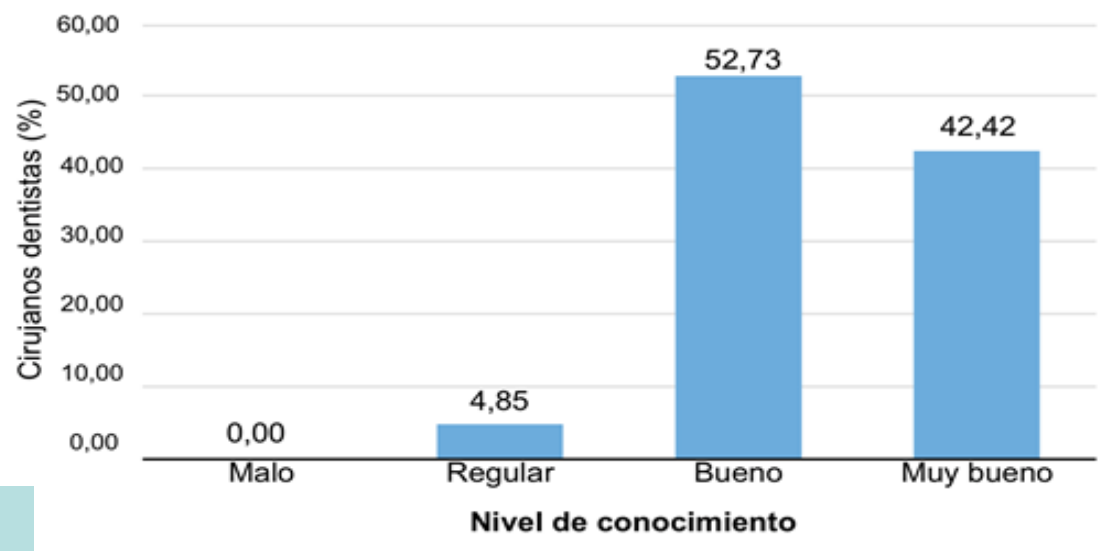


En la Figura 1 se encuentra la distribución de los cirujanos dentistas encuestados según el nivel de conocimiento sobre el manejo de la atención estomatológica por COVID-19. Se observa que 70 encuestados presentaron un nivel muy bueno con $42.42 \%$; seguidamente, 87 encuestados, un nivel bueno con $52.73 \%$; luego, solo 8 encuestados presentaron un nivel regular, $4.85 \%$; y, finalmente, no se encontró ningún encuestado con un nivel de conocimiento malo $(0.00 \%)$.

\section{Figura 2}

Distribución sobre el nivel de conocimiento del manejo estomatológico por COVID-19 de cirujanos dentistas de Tacna (2020), según sexo

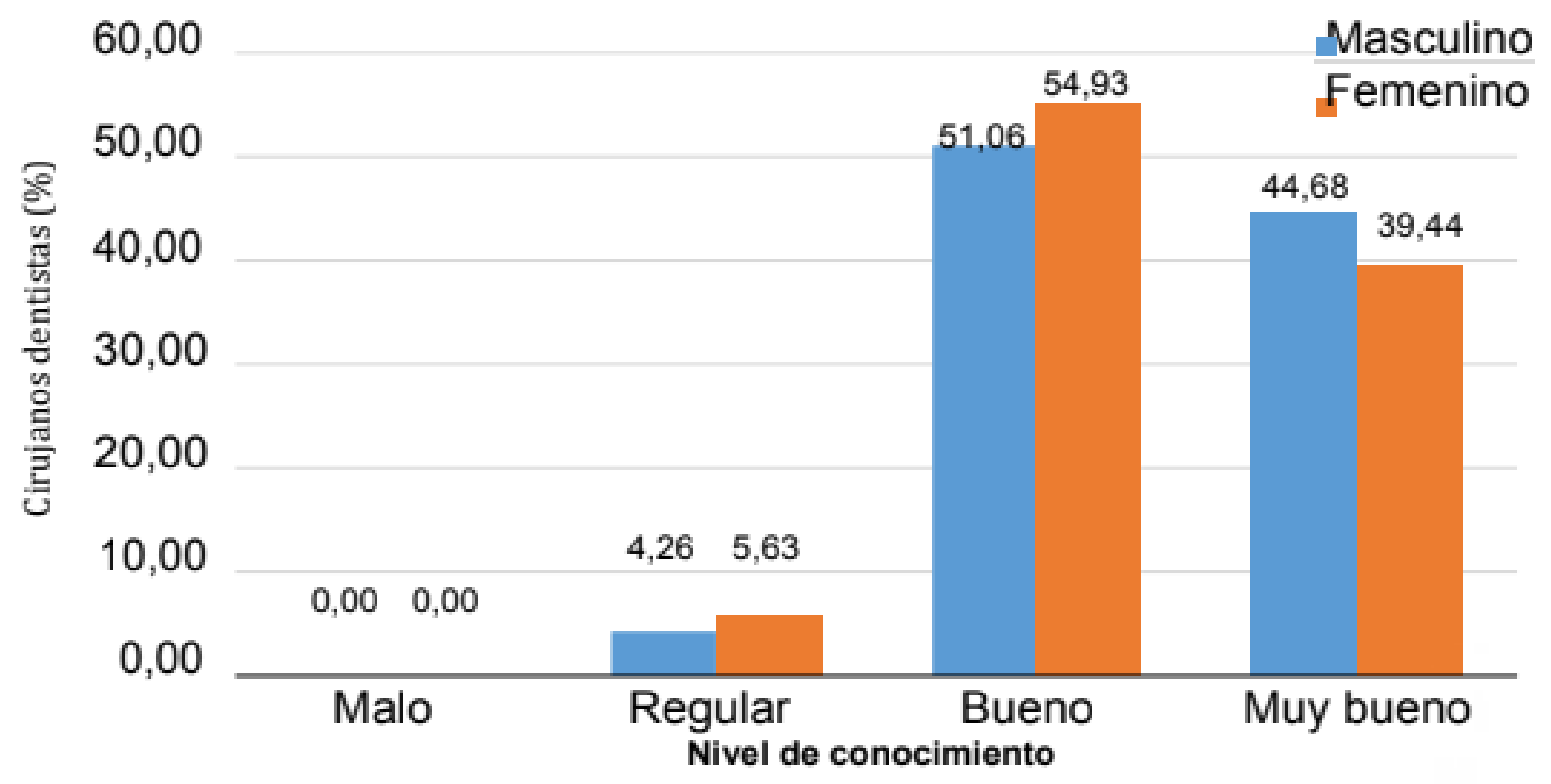

Nota. Fuente:Tabla 2

En la Figura 2 se observa la variable nivel de conocimiento del manejo estomatológico por COVID-19, según sexo de cirujanos dentistas. En cuanto a dentistas del sexo masculino presentaron un nivel muy bueno con $44.68 \%$; seguidamente, presentaron un nivel bueno con $51.06 \%$; luego, presentaron un nivel regular con $4.26 \%$; finalmente, no se encontró ningún encuestado con un nivel de conocimiento malo.

Del mismo modo, en el sexo femenino esta proporción es similar, ya que presentaron un nivel muy bueno con $39.44 \%$; seguidamente, $54.93 \%$ presentaron un nivel bueno; luego, solo $5.63 \%$ presentaron un nivel regular $y$, finalmente, no se encontró ninguna encuestada con un nivel de conocimiento malo. Se puede apreciar que el nivel de conocimiento en el total de los encuestados es similar, tanto en cirujanos dentistas del sexo masculino como femenino. 


\section{Figura 3}

Distribución sobre el nivel de conocimiento del manejo estomatológico por COVID-19 de cirujanos dentistas de Tacna (2020), según edad

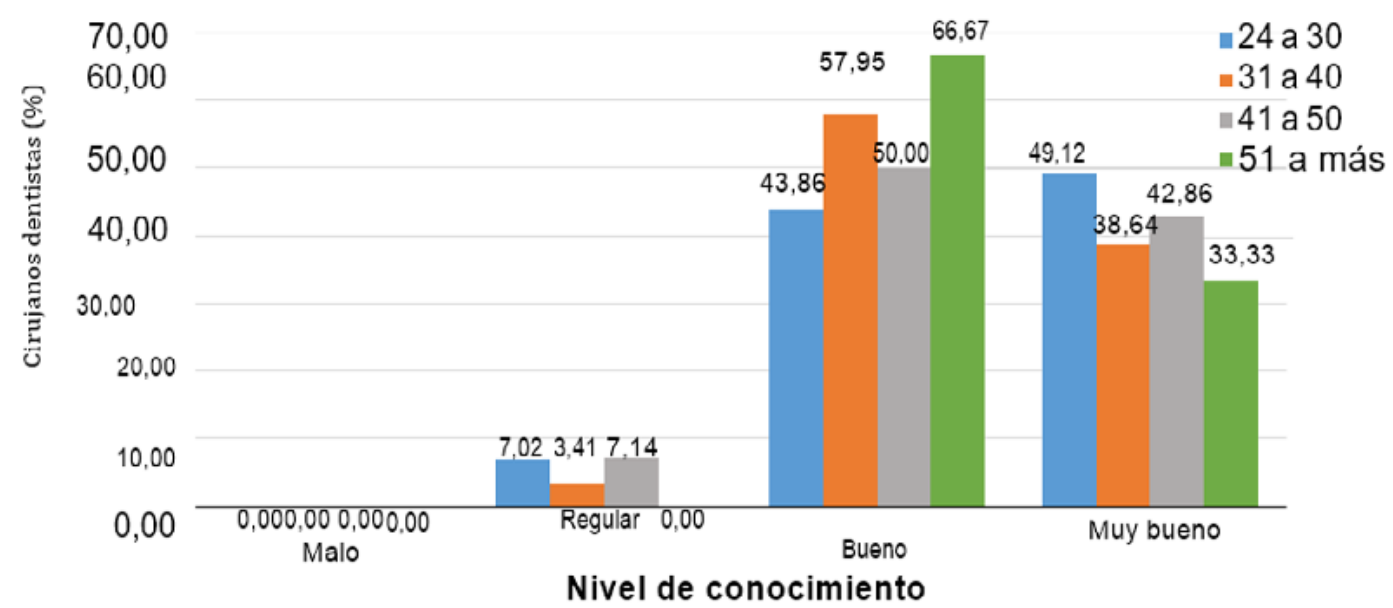

Nota. Fuente: Tabla 3

En la Figura 3 se observa la variable nivel de conocimiento del manejo estomatológico por COVID-19 según edad de cirujanos dentistas. En cuanto a dentistas con edades de 24 a 30 años se presentó un nivel muy bueno con $49.12 \%$; seguidamente, un $43.86 \%$ presentaron un nivel bueno; luego, solo $7.02 \%$ presentaron un nivel regular y, finalmente, no se encontró ningún encuestado con un nivel de conocimiento malo.

Así mismo, solo en cirujanos dentistas con edades de 31 a 40 años se presentó un nivel muy bueno con $38.64 \%$; seguidamente, $57.95 \%$ presentaron un nivel bueno; luego, solo $3.41 \%$ presentaron un nivel regular y, finalmente, no se encontró ningún encuestado con un nivel de conocimiento malo. Seguidamente, solo en cirujanos dentistas con edades de 41 a 50 años esta proporción es similar; presentaron un nivel muy bueno $42.86 \%$; el $50.00 \%$ presentó un nivel bueno y, solo, $7.14 \%$ presentó un nivel regular. Finalmente, no se encontró ningún encuestado con un nivel de conocimiento malo. Por último, solo en cirujanos dentistas con edades de 51 a más años, el $33.33 \%$ presentaron un nivel muy bueno; el $66.67 \%$ presentó un nivel bueno. No se encontró ningún encuestado con un nivel de conocimiento regular o con un nivel de conocimiento malo. Se puede apreciar que el nivel de conocimiento en el total de los encuestados es similar, tanto en cirujanos dentistas con edades de 24 a 30 años, 31 a 40 años, 41 a 50 años y 51 a 79 años.

\section{Discusión}

Nuestros resultados concuerdan con los estudios de Kamate SK et al. en su estudio multinacional, donde se obtuvieron puntuaciones de conocimiento Alto/Bueno con $92.7 \%$. Khader $Y$ et al. nos dicen que los dentistas jordanos conocían los síntomas de la COVID-19, el modo de transmisión, los controles y medidas de infección en las clínicas dentales. Putrino A et al. concluyen que los dentistas italianos presentaron un buen nivel de conocimiento sobre el coronavirus y las medidas de precaución adicionales. Al-Khalifa KS et al., en su estudio, nos dicen que la mayoría de los encuestados presentaron un nivel de conocimiento bueno sobre las preguntas del conocimiento, la práctica y la actitud de los dentistas hacia la pandemia por COVID-19. A diferencia de los resultados del estudio de Kanaparthi A et al. que nos indica que el nivel de conocimiento que predominó fue un nivel moderado con $43.4 \%$. Así también en el estudio de Arora $S$ et al. se obtiene como resultados que el nivel de conocimiento regular fue el que prevaleció con $80.8 \%$.

Por otra parte, respecto al conocimiento sobre manejo estomatológico por COVID-19, según sexo de los cirujanos dentistas (Figura 2), se observa que en el sexo masculino y femenino predomina un nivel de conocimiento bueno con $51.06 \%$ y $54.93 \%$, respectivamente; demostrándose que no existe diferencia estadísticamente significativa en el nivel de conocimiento según el sexo. 
Nuestros resultados concuerdan con los estudios de Al Khalifa KS et al., en donde el sexo no mostró diferencia estadísticamente significativa en el conocimiento, la práctica y la actitud de los dentistas hacia la COVID-19; sin embargo, existen estudios que difieren con nuestro resultado, como el de Putrino $A$ et al., en donde concluyen que sí existe una diferencia estadísticamente significativa entre hombres y mujeres, el cual afirma que los dentistas masculinos están mejor informados sobre el coronavirus, a diferencia de las colegas femeninas que tienen una opinión más cautelosa sobre el tema. Por el contrario, los resultados de los estudios de Arora S et al., Kanaparthi A et al. y Khader Y et al. nos indican que el género de los dentistas mostró una asociación significativa con el nivel de conocimiento, siendo las dentistas femeninas quienes poseen un conocimiento significativamente mayor en comparación con sus homólogos masculinos.

En cuanto al nivel de conocimiento sobre el manejo estomatológico por COVID-19, según edad de los cirujanos dentistas, se observa que en edades de 24 a 30 años predomina un nivel muy bueno con $49.12 \%$; en el grupo de edades de 31 a 40 años predomina un nivel bueno con $57.95 \%$; en el grupo de edades de 41 a 50 años predomina un nivel bueno con $50.00 \%$ y, finalmente, en el grupo de edades de 51 años a más, predomina un nivel bueno con $66.67 \%$, demostrándose que no existe diferencia estadísticamente significativa entre los distintos grupos de edad.

Por el contrario, nuestros resultados difieren con el estudio de AI Khalifa KS et al., donde concluyen que la edad de los participantes tuvo un efecto en el conocimiento sobre COVID-19. Con $90.2 \%$ los participantes mayores ( $\geq 45$ años) estaban más al tanto de los últimos recursos de salud en línea para COVID-19. De la misma forma los resultados de Putrino $\mathrm{A}$. et al. nos indican que, en cuanto a la edad, surgen algunas diferencias entre grupos etarios, siendo los dentistas de 46 y 60 años mejor informados en comparación con colegas más jóvenes.

\section{Conclusiones}

El nivel de conocimiento sobre el manejo estomatológico por COVID-19 en los cirujanos dentistas de Tacna en el año 2020 es bueno con $52.73 \%$, seguido por el nivel muy bueno con $42.42 \%$, y luego el nivel regular con $4.85 \%$. No se encontró ningún encuestado con nivel de conocimiento malo.

No existen diferencias estadísticamente significativas en el nivel de conocimiento según sexo y grupos de edad en los cirujanos dentistas.

\section{Referencias}

1. Yanapa S. Recomendaciones para la atención odontológica durante la pandemia de COVID-19. Revista Odontológica Basadrina, 2020; 4(1):59-64.

2. Colegio Odontológico del Perú. Protocolo de Bioseguridad para el cirujano dentista durante y post pandemia COVID-19. [internet]. Lima: [citado 2020 junio 14]. Disponible en: http://www.cop.org.pe/ wp-content/uploads/2020/04/protocolo-de-bioseguridad-para- el-cirujano-dentista.pdf

3. Kanaparthi A, Dukkireddy D, Gopalaiah H, Kesary SPR, Katne T, Gantala R. Awareness of COVID 19 pandemic among dental practioners of Telangana state, India: A cross sectional surveY. J Oral Biol Craniofac Res. 2020 Oct-Dec; 10(4): 484-489.

4. MINSA. Manejo de la atención estomatológica en el contexto de la pandemia por COVID-19. Directiva Sanitaria. Lima: Ministerio de Salud. Lima:2020.

5. Gaceta Dental. EI COVID-19 y la consulta dental: información y consejos [Internet]. España: [citado 2020 junio 14]. Disponible en: https://gacetadental.com/2020/03/el-covid-19-y-la-consulta-dental- informacióny-consejos-95967

6. Chávez TM, Castro RC. Desafíos de la odontología frente a la pandemia de COVID-19. International Journal of OdontoStomatology, 2020; 14(3): 325-326.

7. Spagnuolo G, De vito D, Rengo S, Tatulb M. COVID-19 Outbreak: An Overview on Dentistry. International Journal of Enuironmental Research and Public Health, 2020; 17(6):2094.

8. Ramírez VM, Medina SP, Morocho MA. Enfermedad por coronavirus 2019 (COVID-19) y su repercusión en la consulta odontológica: una revisión. Odontología Sanmarquina, 2020; 23(2): 139-146.

9. Centro de epidemiologia y vigilancia de las enfermedades Orales- CEVEO. La odontología en los tiempos del coronavirus COVID-19. Int. J. Interdiscip. Dent., 2020; 13(1): 02 
10. Falcón GB, Falcón G. Medidas para prevenir COVID-19 en el consultorio dental. Int. J. Odontostomat., 2020; 14(4): 468-473.

11. Kamate SK, Sharma S, Thakar S, Srivastava D, Sengupta K, Hadi AJ, et al. Assessing Knowledge, Attitudes and Practices of dental practitioners regarding the COVID-19 pandemic: A multinational study. Dent. Med. Probl., 2020;57(1):11-17.

12. Khader Y, Al Nsour M, Al-Batayneh O, Saadeh R, Bashier H, Alfaqih M, et al. Dentists' Awareness, Perception, and Attitude Regarding COVID-19 and Infection Control: Cross-Sectional Study Among Jordanian Dentists. JMIR Public Health Surveill, 2020; 6(2):e18798.

13. Putrino A, Raso M, Magazzino C, Galluccio G. Coronavirus (COVID-19) in Italy: knowledge, management of patients and clinical experience of Italian dentists during the spread of contagion. BMC Oral Health. 2020; 20: 200.

14. Arora S, Abullais SS, Attar N, Pimpale S, Saitullah ZK, Saluja P, et al. Evaluation of Knowledge and Preparedness Among Indian Dentists During the Current COVID-19 Pandemic: A Cross-Sectional Study. J Multidiscip Healthc. 2020; 13: 841-854.

15. Al-khalifa KS, AISheikh R, Al-Swuailem AS, Alkhalifa MS, Al-Johani MH, Al-Moumen SA, et al. Pandemic preparedness of dentists against coronavirus disease: A Saudi Arabian experience. PLoS One. 2020; 15(8): e0237630.

16. Mueras Ventura J. Nivel de conocimiento y prácticas sobre estimulación temprana de madres escolares de dos instituciones educativas pública y privada de Lima, 2019. Tesis. Lima: UNMSM, Lima; 2020.

17. Coronel Arenas J. Nivel de conocimiento y su relación con las prácticas de medidas de bioseguridad del personal que trabaja en el centro de salud Segunda Jerusalen 2017 Rioja (San Martín). Tesis. Nueva Cajamarca: UCSSNC, Cajamarca; 2017.

18. Wikipedia. Pandemia de COVID-19 [Internet]. 2020.[citado 2020 junio 14]. Disponible en: https:// es.wikipedia.org/wiki/Pandemia_de_COVID-19\#Propagaci\%C3\%B3n_internacional_de_la_ enfermedad.

19. Bermúdez JC, Gaitán FC, Aguilera GL. Manejo del paciente en atención odontológica y bioseguridad del personal durante el brote de coronavirus SARS-CoV-2 (COVID-19). Revista ADM, 2020; 77(2):88- 95

20. Sigua RE, Bernal PJ, Lanata FA, Sánchez RC, Rodríguez CJ, Haidar ZS, et al. COVID-19 y la Odontología: una revisión de las recomendaciones y perspectivas para Latinoamérica. Int. J. Odontostomat, 2020; 14(3)

21. MINSA. Recomendaciones para el uso apropiado de mascarillas y respiradores por el personal de salud en el contexto de la COVID-19, Documento Técnico. Lima: MINSA, Ministerio de Salud; 2020.

22. MINSA. Prevención, diagnóstico y tratamiento de personas afectadas por COVID-19 en el Perú. Documento Técnico. Lima: MINSA, Ministerio de Salud; 2020.

23. Real Academia Española. [Internet]. España: [citado 2020 octubre 10]. Disponible en: https://dle.rae.es/ pandemia

\section{Anexos}

Tabla 2

Distribución sobre el nivel de conocimiento del manejo estomatológico por COVID-19 de cirujanos dentistas de Tacna (2020), según sexo

\begin{tabular}{lcccccc} 
Nivel de conocimiento & \multicolumn{3}{c}{ Sexo } & & \multicolumn{2}{c}{ Total } \\
\cline { 2 - 5 } $\begin{array}{l}\text { sobre el manejo } \\
\text { estomatológico por }\end{array}$ & \multicolumn{2}{c}{ Masculino } & \multicolumn{2}{c}{ Femenino } & & \\
COVID-19 & $\mathrm{N}$ & $\%$ & $\mathrm{~N}$ & $\%$ & $\mathrm{n}$ & $\%$ \\
Malo & 0 & 0.00 & 0 & 0.00 & 0 & 0.00 \\
Regular & 4 & 4.26 & 4 & 5.63 & 8 & 4.85 \\
Bueno & 48 & 51.06 & 39 & 54.93 & 87 & 52.73 \\
Muy bueno & 42 & 44.68 & 28 & 39.44 & 70 & 42.42 \\
Total & 94 & 100.00 & 71 & 100.00 & 165 & 100.00 \\
\hline
\end{tabular}

$\begin{array}{llll}\mathrm{x}^{2}=0.535 & \mathrm{GL}=2 & \mathrm{P} \text { valor }=0.465 & \text { No existe diferencia }\end{array}$

Nota. Fuente: Matriz de sistematización de datos 
En la Tabla 2 se observa la variable nivel de conocimiento del manejo estomatológico por COVID-19, según sexo de cirujanos dentistas. En cuanto a dentistas del sexo masculino presentaron un nivel muy bueno con $44.68 \%$, seguidamente presentaron un nivel bueno con $51.36 \%$, luego presentaron un nivel regular con $4.26 \%$ y finalmente no se encontró ningún encuestado con un nivel de conocimiento malo.

Del mismo modo, en el sexo femenino, esta proporción es similar, ya que presentaron un nivel muy bueno $39.44 \%$; seguidamente, $54.93 \%$, presentaron un nivel bueno; luego, solo $5.63 \%$ presentaron un nivel regular y, finalmente, no se encontró ninguna encuestada con un nivel de conocimiento malo. Finalmente, se puede apreciar que el nivel de conocimiento en el total de los encuestados es similar, tanto en cirujanos dentistas del sexo masculino como femenino.

\section{Tabla 3}

\section{Distribución sobre el nivel de conocimiento del manejo estomatológico por COVID-19 de cirujanos dentistas de Tacna (2020), según edad}

\begin{tabular}{|c|c|c|c|c|c|c|c|c|c|c|}
\hline \multirow{3}{*}{$\begin{array}{l}\text { Nivel de conocimiento } \\
\text { sobre el manejo } \\
\text { estomatológico por } \\
\text { COVID-19 }\end{array}$} & \multicolumn{8}{|c|}{ Edad (años) } & \multirow{2}{*}{\multicolumn{2}{|c|}{ Total }} \\
\hline & \multicolumn{2}{|c|}{24 a 30} & \multicolumn{2}{|c|}{31 a 40} & \multicolumn{2}{|c|}{41 a 50} & \multicolumn{2}{|c|}{51 a más } & & \\
\hline & $N$ & $\%$ & $\mathrm{~N}$ & $\%$ & $\mathrm{~N}$ & $\%$ & $\mathrm{n}$ & $\%$ & $\mathrm{n}$ & $\%$ \\
\hline Malo & 0 & 0.00 & 0 & 0,00 & 0 & 0.00 & 0 & 0.00 & 0 & 0.00 \\
\hline Regular & 4 & 7.02 & 3 & 3.41 & 1 & 7.14 & 0 & 0.00 & 8 & 4.85 \\
\hline Bueno & 25 & 43.86 & 51 & 57.95 & 7 & 50.00 & 4 & 66.67 & 87 & 52.73 \\
\hline Muy bueno & 28 & 49.12 & 34 & 38.64 & 6 & 42.86 & 2 & 33.33 & 70 & 42.42 \\
\hline Total & 57 & 100.00 & 88 & 100.00 & 14 & 100.00 & 6 & 100.00 & 165 & 100.00 \\
\hline
\end{tabular}

Nota. Fuente: Matriz de sistematización de datos

En la Tabla 3 se observa la variable nivel de conocimiento del manejo estomatológico por COVID-19 según edad de cirujanos dentistas. En cuanto a dentistas con edades de 24 a 30 años se presentó un nivel muy bueno con $43.86 \%$; seguidamente un $49.12 \%$ presentaron un nivel bueno; luego, solo $7.02 \%$ presentaron un nivel regular y, finalmente, no se encontró ningún encuestado con un nivel de conocimiento malo.

Así mismo, solo en cirujanos dentistas con edades de 31 a 40 años se presentó un nivel muy bueno con $38.64 \%$; seguidamente, $57,95 \%$ presentaron un nivel bueno; luego, solo $3.41 \%$ presentaron un nivel regular y, finalmente, no se encontró ningún encuestado con un nivel de conocimiento malo.

Seguidamente, solo en cirujanos dentistas con edades de 41 a 50 años esta proporción es similar. Presentaron un nivel muy bueno $42.86 \%$; el $50.00 \%$ presentó un nivel bueno y solo $7.14 \%$ presentó un nivel regular. Finalmente, no se encontró ningún encuestado con un nivel de conocimiento malo.

Por último, solo en cirujanos dentistas con edades de 51 a más años, presentaron un nivel muy bueno el $33.33 \%$, el $66.67 \%$ presentó un nivel bueno. No se encontró ningún encuestado con un nivel de conocimiento regular como también con un nivel de conocimiento malo.

Finalmente, se puede apreciar que el nivel de conocimiento en el total de los encuestados es similar, tanto en cirujanos dentistas con edades de 24 a 30 años, 31 a 40 años, 41 a 50 años y 51 a 79 años.

- Conflicto de intereses: La presente investigación no presenta conflicto de intereses entre los investigadores.

- Fuente de financiamiento: La presente investigación fue financiada por los investigadores. 